\title{
Regular heptagon's intersections circles
}

\author{
Zvonko Čerin \\ Zvonko Čerin started his mathematical education at the University of Zagreb in Croa- \\ tia. He received his Ph.D. in infinite dimensional topology at the Louisiana State Uni- \\ versity in Baton Rouge, USA. Since 1976 he teaches mathematics at the University of \\ Zagreb. His fields of interest include topology, geometry, and mathematical education.
}

\section{Introduction}

The regular heptagon (i.e., the planar regular convex polygon with seven vertices) has not been studied extensively like its cousins the equilateral triangle, the square, the regular pentagon, and the regular hexagon. Perhaps the reason is because this is the regular polygon with the smallest number of vertices that cannot be constructed only with compass and straightedge. The few sporadic known results on regular heptagons were reviewed by Leon Bankoff and Jack Garfunkel 30 years ago in the reference [1].

They first recall the following result by Victor Thébault:

The distance from the midpoint $U$ of the side $A B$ of a regular convex heptagon $A B C D E F G$ inscribed in a circle with center $O$ to the midpoint $V$ of the radius perpendicular to $B C$ and cutting this side, is equal to half the side of a square inscribed in the circle.

In der ebenen Geometrie nimmt das Studium der regelmässigen Vielecke einen herausragenden Platz ein. Speziell dem gleichseitigen Dreieck, dem Quadrat, dem regelmässigen Pentagon oder Hexagon kommt eine besondere Bedeutung zu, da man diese Polygone mit Hilfe von Zirkel und Lineal konstruieren kann und Eigenschaften dieser Vielecke ebenfalls auf elementare Weise nachweisen kann. Wie verhält es sich nun aber mit dem regelmässigen Siebeneck? Dieses ist bekanntlich nicht mit Zirkel und Lineal konstruierbar, also wird es auch erheblich schwieriger sein, Eigenschaften von regelmässigen Siebenecken elementar zu beweisen. Im nachfolgenden Beitrag entdeckt der Autor interessante Eigenschaften eines regelmässigen Heptagons A BC DE F G unter Zuhilfenahme des Computeralgebra-Systems Maple V. Dabei zeigen sich unerwartete Phänomene, z.B. in Bezug auf Inkreis und Ankreis des Dreiecks $A B G$. Die entsprechenden Programme sind in einem Anhang angeführt. 


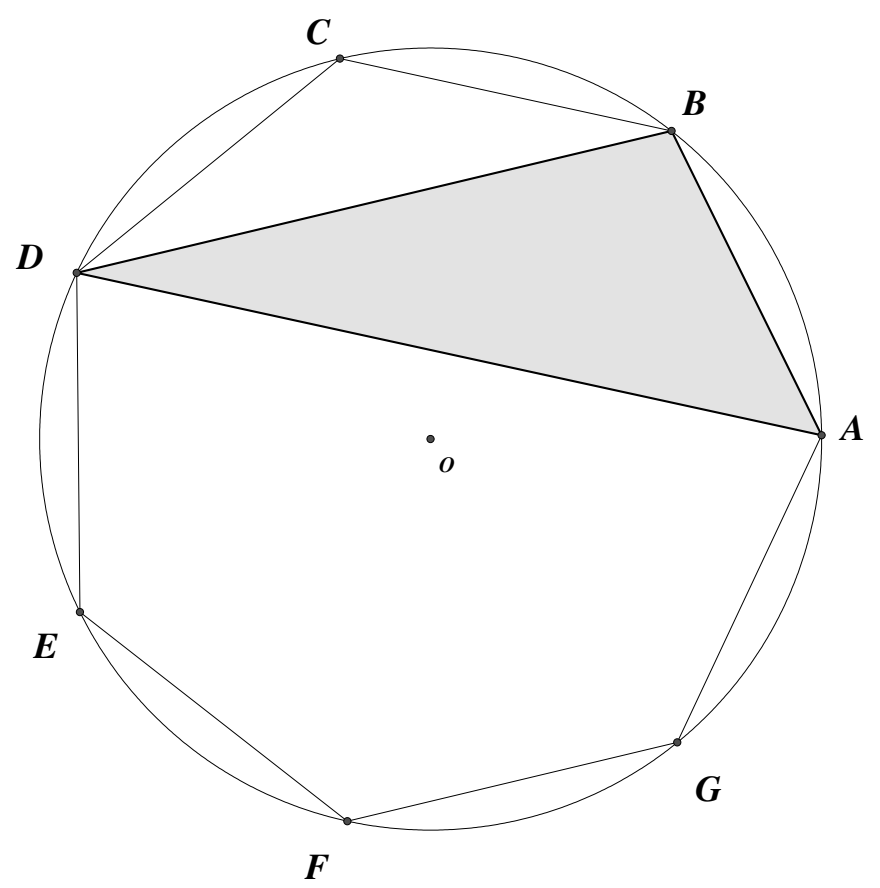

Fig. 1 Regular heptagon $A B C D E F G$ and one of its heptagonal triangles $A B D$
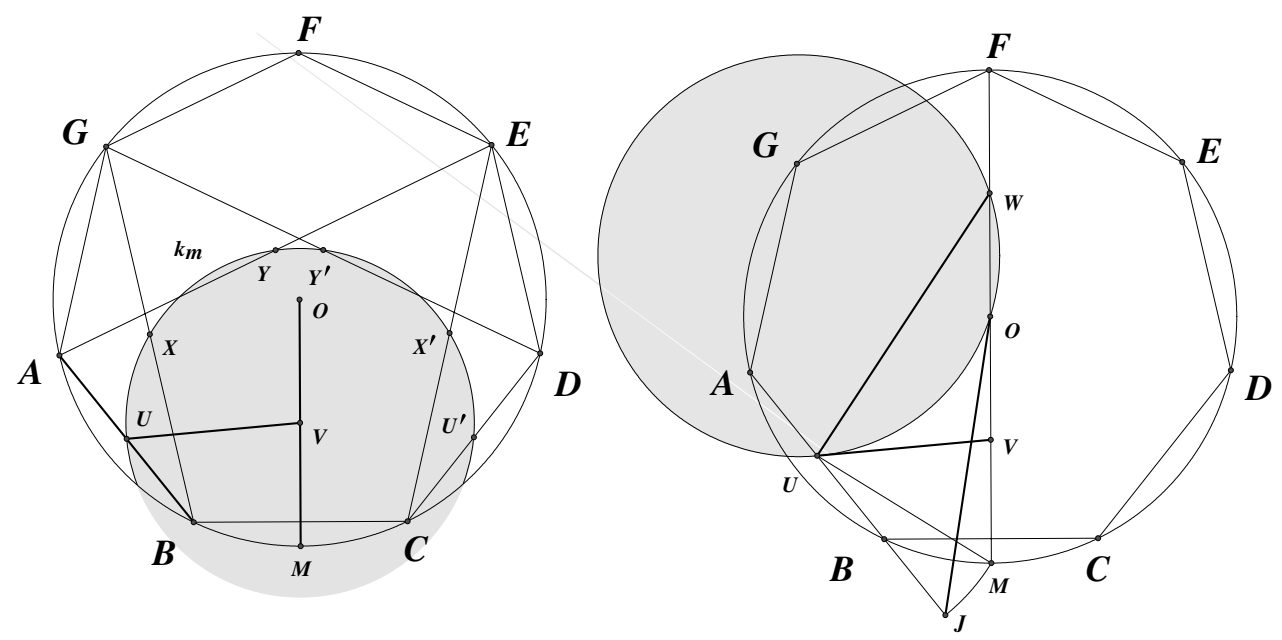

Fig. 2 Illustration of two results by Victor Thébault

In other words, we have $|U V|=\frac{|A O| \sqrt{2}}{2}$. Extending this to diagonals, Hüseyin Demir observed that the circle $k_{m}$ of radius $U V$, centered at $V$, bisects the segments $A B, B G$, $E A, G D, C E$ and $D C$ in the midpoints $U, X, Y, Y^{\prime}, X^{\prime}$ and $U^{\prime}$ (see the left part of Fig. 2). 
The right part of Fig. 2 shows the second result also by Thébault:

If $W$ is the midpoint of $O F, M$ is the point diametrically opposite to $F$ and $J$ is the point on $U B$ produced such that $|U J|=|U M|$, then $|U W|=|U O| \sqrt{2}$, $|O J|=\frac{|A O| \sqrt{6}}{2}$ and the line $U V$ is tangent to the circle through $U, O$ and $W$.

The rest of [1] is a study of the heptagonal triangle (for example, the triangle $A B D$ in Fig. 1) whose angles are $\frac{\pi}{7}, \frac{2 \pi}{7}$ and $\frac{4 \pi}{7}$ radians. We mention only the following four of their properties from an extensive list (see pages 14, 17, and 19 of [1]):

- The sum of cotangents of angles is equal to $\sqrt{7}$.

- The sum of squares of cotangents of angles is equal to 5.

- The triangle formed by joining the feet of the internal angle bisectors of the heptagonal triangle is isosceles.

- The two tangents from the orthocenter to the circumcircle of the heptagonal triangle are mutually perpendicular.

Today we can add new results to the above list with some help from computers. In papers [2], [3], and [4] the author has improved some of the above theorems. We added six more midpoints of segments in Demir's observation that also lie on the circle $k_{m}$ in [2]. Later in [4] we recognized two regular heptagons inscribed in $k_{m}$ whose vertices are these midpoints. The reference [3] contains the improvement of the second Thébault result above and some new geometric relationships in regular heptagons.

In this paper we show that the intersections of many lines associated to a regular heptagon $A B C D E F G$ lie on its interesting circles determined either by incenters or by the excenters of the triangles $D E B$ and $A B G$. In other words, we discover many regular heptagons related to a given regular heptagon which all have easy construction with compass and straightedge.

Recall that every triangle $A B C$ has the incircle and three excircles which touch the lines $B C, C A$ and $A B$. Their centers are the incenter $I$ and the excenters $I_{a}, I_{b}$ and $I_{c}$. The incenter is inside while the excenters are outside the triangle and in the natural order $I_{a}$ is called the first excenter since it lies on the first angle bisector $A I$.

In order to simplify our statements we use the following notation: The parallel and the perpendicular to the line $\ell$ through the point $X$ are $X \| \ell$ and $X \perp \ell$.

In our proofs we shall use complex numbers because they provide simple expressions and arguments. There are several excellent books, for example [7], [5], [9], [6], [10], and [8], that give introductions into the method which we utilize. In an appendix we implement this approach in Maple V. The reader can see there how the intersection of two lines is computed. This is in fact the only thing to learn.

A point $P$ in the Gauss plane is identified with a complex number $P$ (its $a f f i x$ ). The complex conjugate of $P$ is denoted $\bar{P}$. We shall always assume that the complex coordinates of the vertices of the heptagon $A B C D E F G$ are $A=1, B=f^{2}, C=f^{4}, D=f^{6}, E=f^{8}$, $F=f^{10}$, and $G=f^{12}$, where $f$ is a $14^{\text {th }}$ root of unity. Had we used the $7^{\text {th }}$ roots of unity 
some important points like the midpoints $P$ and $Q$ of the shorter $\operatorname{arcs} A G$ and $A B$ would have complicated affices. Hence, all these points are on the unit circle $k$ whose center is the origin $O$.

\section{The first circle from incenters}

We begin our study with the circle $m$ whose center is the incenter $V$ of the triangle $A B G$ and which goes through the incenter $U$ of the triangle $B E D$.

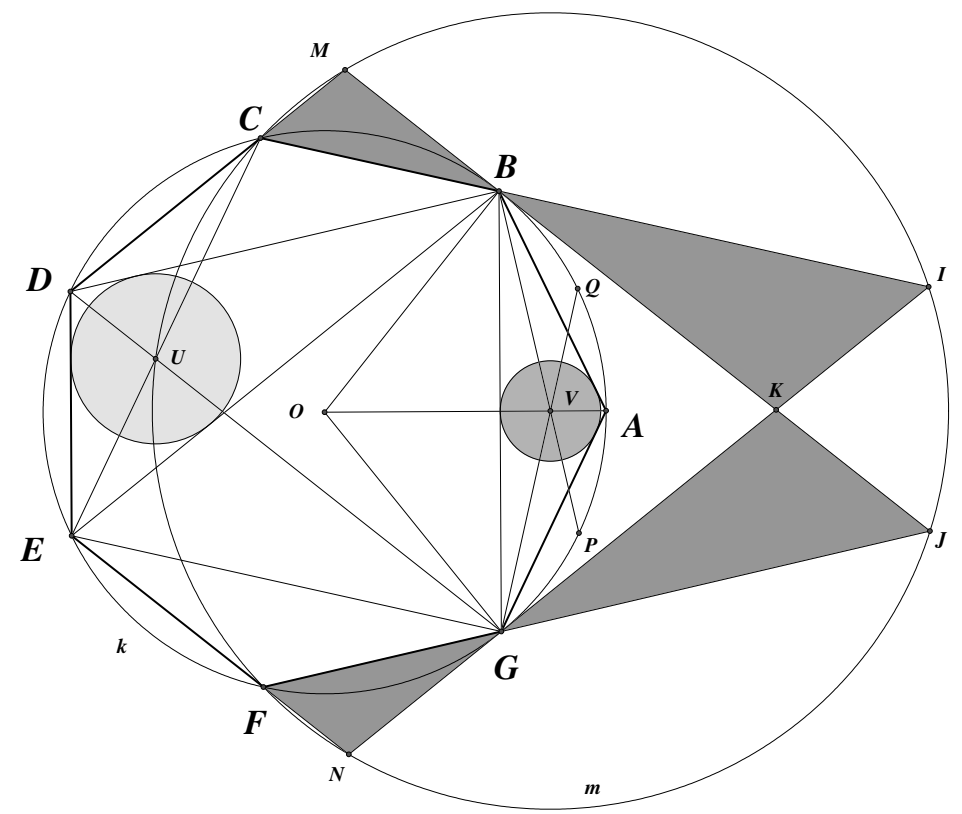

Fig. 3 The circle $m$ with the center at the incenter of $A B G$ and the radius $\sqrt{2}$ has interesting properties (Theorems 1-3)

Theorem 1. The circle $m$ has the radius $\sqrt{2}$ and it goes through the points $C$ and $F$.

Let $K=I N \cap J M$ where the points $I, N$ and $J, M$ are intersections of $B C, E F$ and $F G$, $C D$ with $G \perp G O$ and $B \perp B O$.

Theorem 2. The points $I, J, M$ and $N$ are on the circle $m$ and the point $V$ is the midpoint of the segment $K O$.

Theorem 3. The triangles $B I K, G J K, B C M, F G N$ are heptagonal.

Proof of Theorems 1-3. The points $P$ and $Q$ are $f^{13}$ and $f$. Note that $|B C|=|C D|$ so that $\varangle B E C=\varangle C E D$. It follows that $E C$ is the bisector of the angle $E$ in the triangle $B D E$. In the same way we see that the line $D G$ is the bisector of the angle $D$ in the triangle $B D E$ and that $B P, G Q$ and $A O$ are the bisectors of the angles $B, G$ and $A$ in 
the triangle $A B G$. The incenters $U$ and $V$ are therefore the intersections $C E \cap D G$ and $B P \cap G Q$. Hence, $U=-f^{5}+f^{4}-1$ and $V=f^{2}-f^{3}+f^{4}-f^{5}$. The equation of the circle $m$ with the center $V$ and the radius $\sqrt{2}$ is $(z-V) \overline{(z-V)}=2$ or

$$
z \bar{z}+f^{2}(f-1)\left(f^{2}+1\right)(z+\bar{z})+f^{5}-2 f^{4}+2 f^{3}-f^{2}-1=0 .
$$

When we substitute the coordinates of the points $C, F$, and $U$ for $z$ into this equation we obtain an expression that has the polynomial $p_{-}=f^{6}-f^{5}+f^{4}-f^{3}+f^{2}-f+1$ as a factor. Since $f^{14}-1$ factors as $(f-1)(f+1) p_{-} p_{+}$, with $p_{+}=f^{6}+f^{5}+f^{4}+$ $f^{3}+f^{2}+f+1$ and $p_{+}=1+2 i\left(1+2 \cos \frac{\pi}{7}\right) \sin \frac{2 \pi}{7} \neq 0$, we see that $p_{-}=0$ so that the points $C, F$, and $U$ are on the circle $m$.

In order to find the affix of the point $I$ (the intersection of the line $B C$ with the perpendicular $G \perp G O$ to the line $G O$ in $G$ ) notice that $B C$ has the equation

$$
\left(f^{5}-f^{3}\right) z-\left(f^{4}-f^{2}\right) \bar{z}+f^{5}+f^{2}=0
$$

while the equation of $G \perp G O$ is

$$
f^{2} z-f^{5} \bar{z}-2=0
$$

Now we must solve in $z$ and $\bar{z}$ the system formed by these two equations in order to obtain $I=f+f^{2}-f^{5}$. For the points $J, M$, and $N$ we get similarly

$$
J=\bar{I}, \quad M=f^{4}-f^{3}+f^{2}+f-1, \quad \text { and } \quad N=\bar{M} .
$$

Once we know the points $I, J, M$, and $N$ the rest of the proof is a routine verification. The substitution of their coordinates into the equation of the circle $m$ always contain the factor $p_{-}$which is zero. Notice that the lines $I N$ and $J M$ are tangents of the circle $k$. Finally, solving linear equations we can compute the affix of the intersection $K=2 \mathrm{~V}$ of these tangents. Clearly, the point $V$ is the midpoint of the segment $K O$. Then we look for conditions (see [5] and the appendix) that the triangles $J K G$ and $F N G$ are directly similar to the heptagonal triangle $D E G$ and that the triangles $I K B$ and $C M B$ are reversely similar to the heptagonal triangle $D E G$. In all four cases the above factor $p_{-}$of $f^{14}-1$ (which is zero) appears.

\section{Three regular heptagons inscribed in $m$}

In the next two theorems we shall describe three regular heptagons inscribed in the circle $m$ whose easy constructions with compass and straightedge depend on the points $I, J, M$ and $N$.

Theorem 4. Let the points $H, J^{\prime}, S, U^{\prime}, H^{\prime}, I^{\prime}, S^{\prime}$ be intersections of $A P, A C, C G$, $B E, B F, A F, D G$ with $B E, N\|F G, K\| A G, M\|C E, K\| C G, K\|B F, M\| C G$, respectively. Then $F U M H I J^{\prime} S$ and $N U^{\prime} C H^{\prime} I^{\prime} J S^{\prime}$ are regular heptagons inscribed in $m$ (see Fig. 4). The point $H$ lies also on $N \| B F$ and $U^{\prime}$ is the incenter of the triangle $D E G$. 


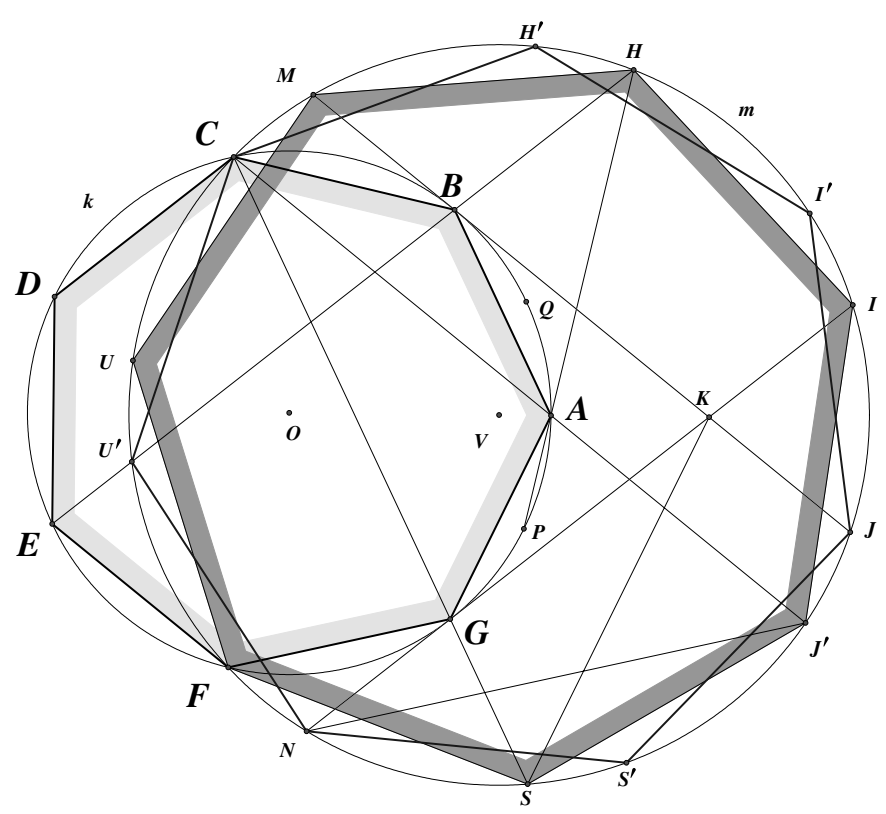

Fig. 4 The regular heptagons $F U M H I J^{\prime} S$ and $N U^{\prime} C H^{\prime} I^{\prime} J S^{\prime}$ inscribed in the circle $m$ (Theorem 4)

Theorem 5. The midpoints $B_{0}, A_{0}, G_{0}, F_{0}, E_{0}, D_{0}$, and $C_{0}$ of the shorter arcs $N F, U^{\prime} U$, $C M, H H^{\prime}, I^{\prime} I, J J^{\prime}$, and $S S^{\prime}$ are vertices of a regular heptagon whose sides are parallel to the corresponding sides of BAGFEDC (see Fig. 5).

Proof of Theorems 4 and 5. The equations of the lines $A P$ and $B E$ are

$$
(1-f) z+\left(f^{13}-1\right) \bar{z}+f-f^{13}=0
$$

and

$$
\left(f^{12}-f^{6}\right) z+\left(f^{8}-f^{2}\right) \bar{z}+f^{8}-f^{20}=0 .
$$

Their intersection $H$ is $-f^{5}+2 f^{4}-f^{3}+2 f^{2}-f+1$. Also,

$$
\begin{aligned}
J^{\prime} & =-2 f^{5}+2 f^{4}-2 f^{3}+f^{2}+1, & S & =-2 f^{5}+f^{4}-2 f^{3}+2 f^{2}-f, \\
I^{\prime} & =-f^{5}+2 f^{4}-2 f^{3}+2 f^{2}+1, & H^{\prime} & =-f^{5}+f^{4}+f^{2}+f-1, \\
U^{\prime} & =-f^{3}+f^{2}-1, & S^{\prime} & =-f^{5}-f^{3}+f .
\end{aligned}
$$

Let us define the number $w$ to be $f-f^{2}-f^{4}$. Then $\left|S^{\prime} V\right|^{2}=\left(S^{\prime}-V\right)\left(\overline{S^{\prime}}-\bar{V}\right)=$ $w(1-w)$ is equal to 2 . In the same way we verify that $|H V|^{2},\left|J^{\prime} V\right|^{2},|S V|^{2},\left|U^{\prime} V\right|^{2}$, $\left|H^{\prime} V\right|^{2}$, and $\left|I^{\prime} V\right|^{2}$ are also 2 so that the heptagons $F U M H I J^{\prime} S$ and $N U^{\prime} C H^{\prime} I^{\prime} J S^{\prime}$ are inscribed in $m$. That these are regular heptagons follows from the fact that $|F U|^{2},|U M|^{2}$, $|M H|^{2},|H I|^{2},\left|I J^{\prime}\right|^{2},\left|J^{\prime} S\right|^{2},|S F|^{2},\left|N U^{\prime}\right|^{2},\left|U^{\prime} C\right|^{2},\left|C H^{\prime}\right|^{2},\left|H^{\prime} I^{\prime}\right|^{2},\left|I^{\prime} J\right|^{2},\left|J S^{\prime}\right|^{2}$, and $\left|S^{\prime} N\right|^{2}$ all have the same value $2 f^{5}-2 f^{2}+4$. 


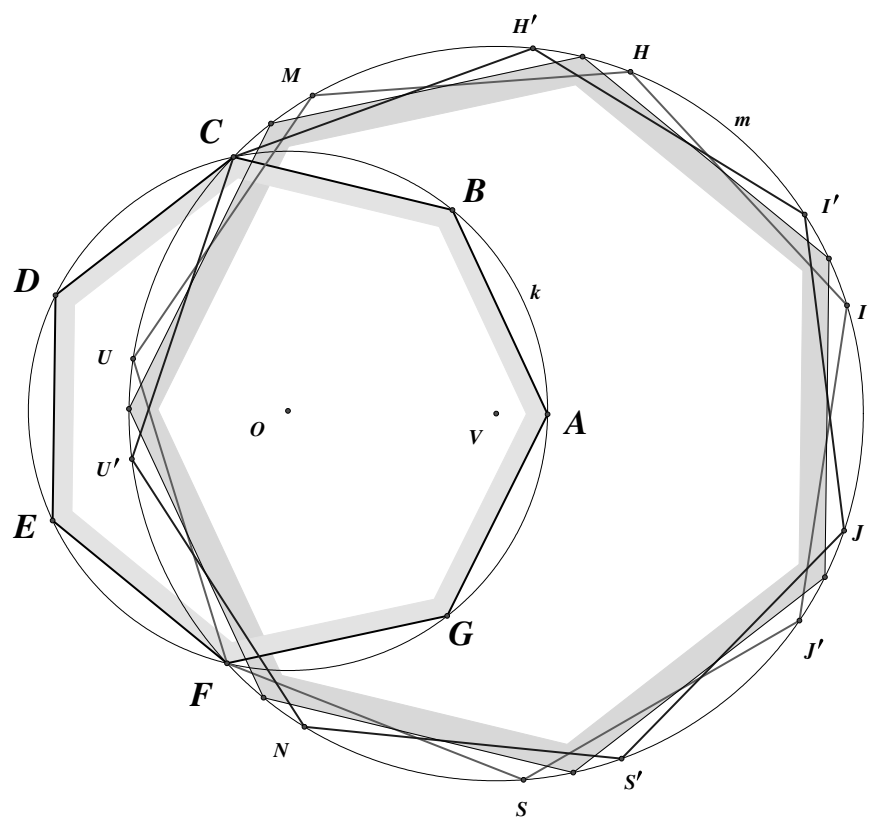

Fig. 5 The regular heptagon on midpoints of shorter arcs $N F, U^{\prime} U, C M, H H^{\prime}, I^{\prime} I, J J^{\prime}$, and $S S^{\prime}$ has sides parallel to the corresponding sides of $B A G F E D C$ (Theorem 5)

In order to find the midpoint $B_{0}$ of the shorter arc $F N$ we use that it has equal distances from the points $F$ and $N$, that it lies on $m$, that its distance to the point $F$ is less than $\sqrt{2}$ (the radius of $m$ ) and that it is a polynomial of order at most five in $f$. Hence, $B_{0}=-f^{5}+f^{4}-f^{3}+(1-\sqrt{2}) f^{2}$. Similarly,

$$
\begin{array}{ll}
A_{0}=-f^{5}+f^{4}-f^{3}+f^{2}-\sqrt{2}, & C_{0}=-f^{5}-(1-\sqrt{2}) f^{4}-f^{3}+f^{2}, \\
D_{0}=(1+\sqrt{2})(1-f)\left(f^{4}+f^{2}+2-\sqrt{2}\right), & E_{0}=\overline{D_{0}}, \quad F_{0}=\overline{C_{0}}, \quad G_{0}=\overline{B_{0}} .
\end{array}
$$

It is now easy to check that the regular heptagons $B_{0} A_{0} G_{0} F_{0} E_{0} D_{0} C_{0}$ and $B A G F E D C$ have parallel corresponding sides.

\section{Four inscribed regular heptagons}

In this section we describe four regular heptagons inscribed in the circumcircles of the triangles $B I K, G J K, F G N$ and $B C M$ and show that their centers are vertices of a rectangle.

Theorem 6. Let $D_{1}, C_{1}, B_{1}, G_{1}, G_{2}, D_{2}, C_{2}, B_{2}, B_{3}, G_{3}, F_{3}, E_{3}, A_{4}, E_{4}, D_{4}, C_{4}$ be intersections of $E G, B F, C G, A G, A G, E G, D G, C G, A B, B F, B E, B D, A B, B D, B E$, $B F$ with $C F, D G, A F, F\|B C, K\| F G, J\|D E, J\| B F, N\|B C, K\| B C, I \| E F$, $I\|A B, I\| B G, C \| F G, C F, C G, A C$. Then $N F D_{1} C_{1} B_{1} G G_{1}, G G_{2} K J D_{2} C_{2} B_{2}$, 


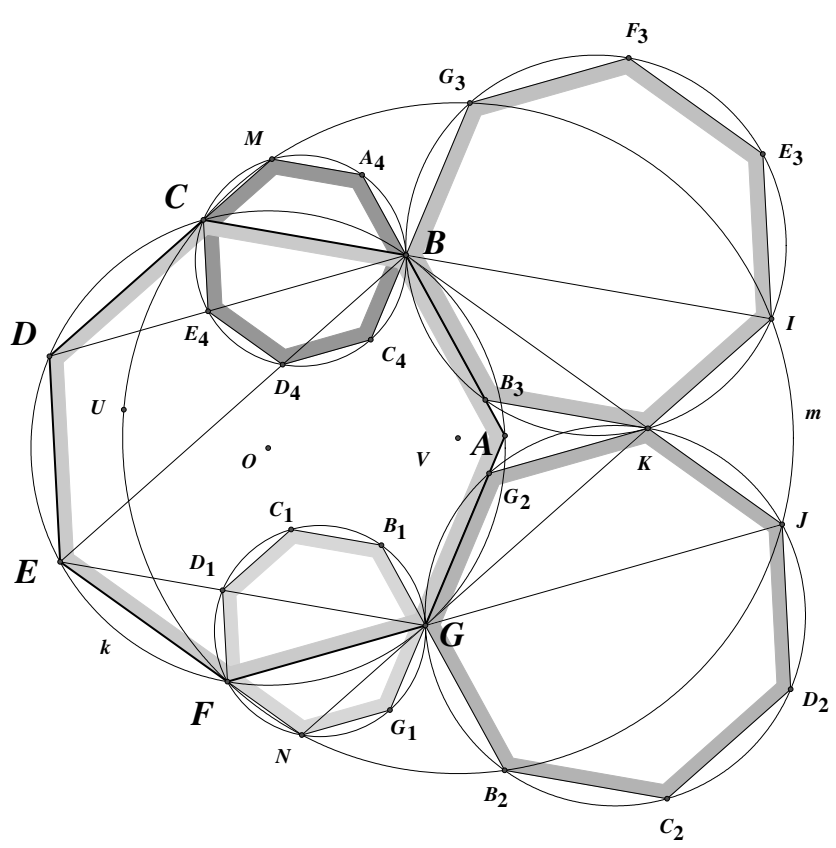

Fig. 6 Four regular heptagons inscribed in circumcircles of the triangles $F G N, G J K, B I K, B C M$ (Theorem 6)

I $K B_{3} B G_{3} F_{3} E_{3}$, and $B A_{4} M C E_{4} D_{4} C_{4}$ are regular heptagons inscribed in the circumcircles of $F G N, G J K, B I K$, and $B C M$ whose sides are parallel to the corresponding sides of FEDCBAG, AGFEDCB, DCBAGFE, and BAGFEDC (see Fig. 6).

Proof. The circumcenter $O_{1}$ of the triangle $F G N$ is $-f^{5}+f^{4}-f^{3}$ and the equation of its circumcircle $m_{1}$ is

$$
\left(f^{4}+f^{2}+1\right) z \bar{z}-f^{4}\left(f^{2}+1\right)\left(z+f^{8} \bar{z}\right)+f^{16}=0 .
$$

The points $D_{1}, C_{1}, B_{1}, G_{1}$ are $-f^{5}+f^{4}-f^{2},-f^{5}+f^{4}-f^{3}+f-1,-f^{5}+2 f^{4}-2 f^{3}+$ $f^{2}-f+1,-f^{5}+f^{4}-f^{3}-f^{2}+f$, respectively. As the expression $f^{2 n}\left(N-O_{1}\right)+O_{1}$, for $n=1, \ldots, 6$ is $G_{1}, G, B_{1}, C_{1}, D_{1}$, and $F$, we infer that $N F D_{1} C_{1} B_{1} G G_{1}$ is a regular heptagon inscribed in $m_{1}$. That its sides are parallel with the corresponding sides of the heptagon $F E D C B A G$ is now easy to verify. The remaining three circumcircles of the triangles $G J K, B I K$, and $B C M$ are treated similarly.

Theorem 7. The circumcenters $\mathrm{O}_{1}, \mathrm{O}_{2}, \mathrm{O}_{3}, \mathrm{O}_{4}$ of the triangles $\mathrm{FGN}, \mathrm{GJK}, \mathrm{BIK}$, and $B C M$ are vertices of a rectangle - the translation for the vector $\overrightarrow{O V}$ of the rectangle $P_{1} G B Q_{1}$ where $P_{1}$ and $Q_{1}$ are the midpoints of the shorter arcs $E F$ and $C D$ (see Fig. 7).

Proof. Notice that $P_{1}=f^{9}, Q_{1}=f^{5}, O_{2}=-2 f^{5}+f^{4}-f^{3}+f^{2}, O_{3}=-f^{5}+f^{4}$ $f^{3}+2 f^{2}$ and $O_{4}=f^{4}-f^{2}+f^{2}$. The claim follows from $P_{1}+V=O_{1}, G+V=O_{2}$, $B+V=O_{3}$ and $Q_{1}+V=O_{4}$. 


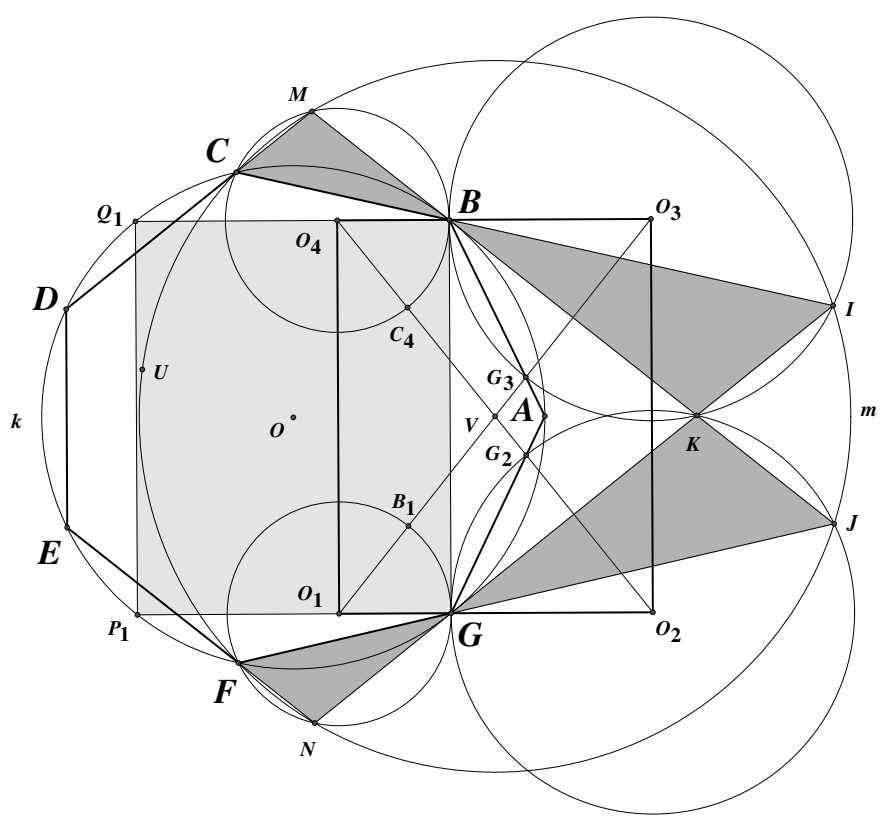

Fig. 7 The circumcenters of the triangles $F G N, G J K$, $B I K, B C M$ are vertices of a rectangle (Theorem 7)

\section{The second circle from excenters}

Since the circle $m$ is determined by the incenters of the triangles $D E B$ and $A B G$, we can ask if the excenters of these triangles give a circle containing intersections of some lines related to the regular heptagon $A B C D E F G$. The answer to this natural question is given in the following theorems.

Theorem 8. Let $U_{0}$ and $V_{0}$ be the first excenters of the triangles $D E B$ and $A B G$ in the regular heptagon $A B C D E F G$ inscribed to the circle $k$ with the center $O$ and the radius $R$. Then the circle $n$ with the center $V_{0}$ and the radius $\frac{R \sqrt{2} \cos \frac{3 \pi}{14}}{\sin \frac{\pi}{14}}$ goes through the points $U_{0}, I$ and $J$ (see Fig. 8).

Proof. Since the excenter $V_{0}$ is the intersection of lines $A O$ and $G \perp G V$ we get

$$
V_{0}=f^{5}-f^{4}+f^{3}-f^{2}-2
$$

Similarly, the excenter $U_{0}$ is the intersection of the lines $D G$ and $E \perp E U$ so that $U_{0}=$ $-f^{5}-f^{4}+1$. The equation of the circle $n$ with the center at the point $V_{0}$ through the point $U_{0}$ is $z \bar{z}-V_{0}(z+\bar{z})+7 f^{5}-2 f^{4}+2 f^{3}-7 f^{2}-9=0$. Its radius is $\sqrt{14-10 f^{5}+4 f^{4}-4 f^{3}+10 f^{2}}$ which reduces to $\frac{\sqrt{2} \cos \frac{3 \pi}{14}}{\sin \frac{\pi}{14}}$. By substitution of coordinates of the points $I$ and $J$ in the above equation we can verify that they lie on the circle $n$. 


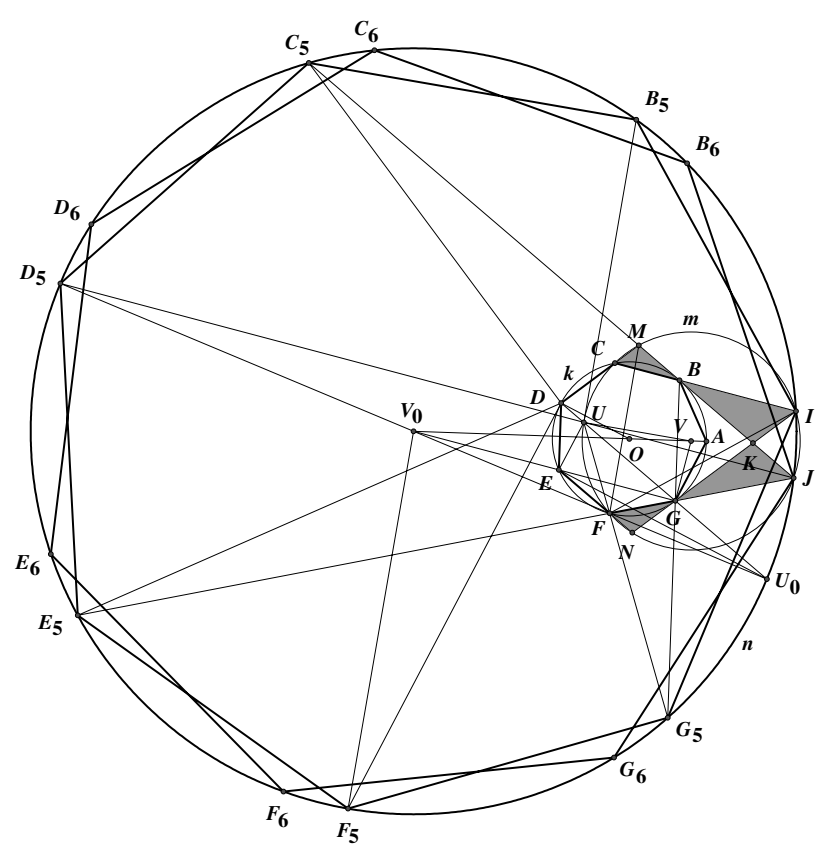

Fig. 8 The circle $n$ determined by excenters $U_{0}$ and $V_{0}$ and two regular heptagons inscribed in it (Theorems 8 and 9)

Theorem 9. Let the points $G_{5}, F_{5}, E_{5}, D_{5}, C_{5}, B_{5}, G, F_{6}, E_{6}, D_{6}, C_{6}, B_{6}$ be intersections of $B G, D \perp D O, F G, J U, J M, I \perp F I, J \perp C J, I N, C V_{0}, J V, E \perp E O, B G$ with $F U, V_{0}\|F M, D\| B U, U_{0}\|I M, D \perp C D, U \perp U V, E\| B P, E \perp E F, I \| A E$, $E \perp C E, J \| M Q, D \perp D O$. Then $I G_{5} F_{5} E_{5} D_{5} C_{5} B_{5}$ and $J G_{6} F_{6} E_{6} D_{6} C_{6} B_{6}$ are regular heptagons inscribed in $n$ (see Fig. 8). The midpoints $A_{7}, G_{7}, F_{7}, E_{7}, D_{7}, C_{7}, B_{7}$ of the shorter arcs $I J, G_{5} G_{6}, F_{5} F_{6}, E_{5} E_{6}, D_{5} D_{6}, C_{5} C_{6}, B_{5} B_{6}$ are vertices of a regular heptagon whose sides are parallel to the corresponding sides of AG FEDCB (see Fig. 10).

Proof. Solving linear equations we get $G_{5}=-2 f^{5}-2 f^{3}+f^{2}-2 f+1, F_{5}=-3 f^{4}-$ $2 f^{2}-f-2, E_{5}=2 f^{5}-2 f^{4}+f^{3}-4 f^{2}-4, D_{5}=3 f^{5}-f^{4}+2 f^{3}-2 f^{2}-5$, $C_{5}=4 f^{5}-f^{4}+3 f^{3}-f^{2}+f-3, B_{5}=f^{5}+3 f^{3}+f-1, G_{6}=\overline{B_{5}}, F_{6}=\overline{C_{5}}$, $E_{6}=\overline{D_{5}}, D_{6}=\overline{E_{5}}, C_{6}=\overline{F_{5}}$ and $B_{6}=\overline{G_{5}}$. Since the expressions $f^{2 k}\left(I-V_{0}\right)+V_{0}$ and $f^{2 k}\left(J-V_{0}\right)+V_{0}$ for $k$ from 1 to 6 are $B_{5}, C_{5}, D_{5}, E_{5}, F_{5}, G_{5}$ and $B_{6}, C_{6}, D_{6}, E_{6}$, $F_{6}, G_{6}$ we conclude that $I G_{5} F_{5} E_{5} D_{5} C_{5} B_{5}$ and $J G_{6} F_{6} E_{6} D_{6} C_{6} B_{6}$ are regular heptagons inscribed in $n$.

Let $\eta=\frac{\sqrt{14}}{7}$. As in the proof of Theorem 5 we find

$$
\begin{gathered}
A_{7}=(1-3 \eta)\left(f^{5}+\frac{1+14 \eta}{11} f^{3}(f-1)-f^{2}-2\right), \\
B_{7}=f^{5}-(1-2 \eta) f^{4}+(1+\eta) f^{3}-(1-5 \eta) f^{2}+\eta f-2(1-\eta) .
\end{gathered}
$$


The condition for the lines $A B$ and $A_{7} B_{7}$ to be parallel (which must be zero) holds because it contains $p_{-}$as a factor. Since $A_{7} B_{7} C_{7} D_{7} E_{7} F_{7} G_{7}$ is obviously a regular heptagon it follows that its sides are parallel with the corresponding sides of $A B C D E F G$.

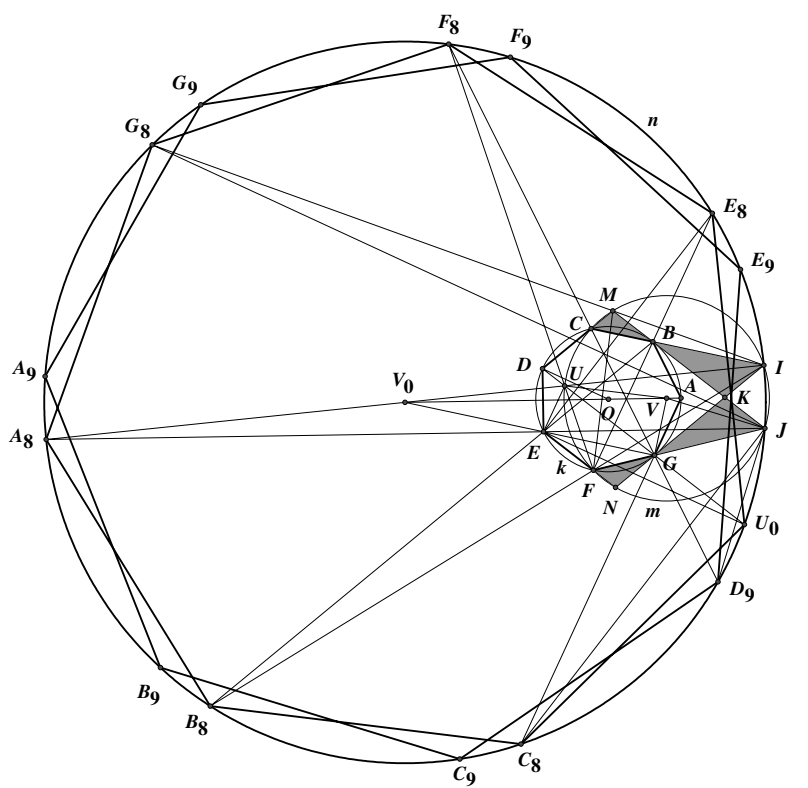

Fig. 9 Another two easily constructible regular heptagons inscribed in the circle $n$ (Theorem 10)

Theorem 10. Let the points $C_{8}, B_{8}, A_{8}, G_{8}, F_{8}, E_{8}, D_{9}, C_{9}, B_{9}, A_{9}, G_{9}, F_{9}, E_{9}$ be intersections of $A G, B E, E J, I M, C G, B F, C G, B F, J N, D I, D G, A B, B E$ with $J \perp J M$, $F I, I V_{0}, J\|C Q, F U, E \perp E F, J \perp J V, D\| F O, U_{0}\left\|B D, J V_{0}, C J, N\right\| C O, C V_{0}$. Then $U_{0} C_{8} B_{8} A_{8} G_{8} F_{8} E_{8}$ and $D_{9} C_{9} B_{9} A_{9} G_{9} F_{9} E_{9}$ are regular heptagons inscribed in $n$ (see Fig. 9). The midpoints $D_{10}, C_{10}, B_{10}, A_{10}, G_{10}, F_{10}, E_{10}$ of the shorter arcs $U_{0} D_{9}$, $C_{8} C_{9}, B_{8} B_{9}, A_{8} A_{9}, G_{8} G_{9}, F_{8} F_{9}, E_{8} E_{9}$ are vertices of a regular heptagon whose sides are parallel to the corresponding sides of DCBAGFE (see Fig. 10).

Proof. From the linear equations we get $C_{8}=-2 f^{5}-f^{4}-f^{3}-f^{2}-f-1, B_{8}=$ $f^{5}-2 f^{4}-f^{3}-2 f^{2}-f-3, A_{8}=3 f^{5}-2 f^{4}+2 f^{3}-3 f^{2}-f-4, G_{8}=$ $4 f^{5}-2 f^{4}+4 f^{3}-3 f^{2}+2 f-5, F_{8}=2 f^{5}+f^{4}+2 f^{3}+f-2, E_{8}=f^{3}+2 f^{2}$, $D_{9}=\overline{E_{8}}, C_{9}=\overline{F_{8}}, B_{9}=\overline{G_{8}}, A_{9}=\overline{A_{8}}, G_{9}=\overline{B_{8}}, F_{9}=\overline{C_{8}}$ and $E_{9}=\overline{U_{0}}$. Since $f^{2 k}\left(U_{0}-V_{0}\right)+V_{0}$ and $f^{2 k}\left(D_{9}-V_{0}\right)+V_{0}$ for $k$ from 1 to 6 are $E_{8}, F_{8}, G_{8}, A_{8}, B_{8}, C_{8}$ and $E_{9}, F_{9}, G_{9}, A_{9}, B_{9}, C_{9}$ we conclude that $U_{0} C_{8} B_{8} A_{8} G_{8} F_{8} E_{8}$ and $D_{9} C_{9} B_{9} A_{9} G_{9} F_{9} E_{9}$ are regular heptagons inscribed in $n$.

This time we get

$$
A_{10}=(1+3 \eta)\left(f^{5}+\frac{1-14 \eta}{11} f^{3}(f-1)-f^{2}-2\right)
$$




$$
B_{10}=f^{5}-(1+2 \eta) f^{4}+(1-\eta) f^{3}-(1+5 \eta) f^{2}-\eta f-2(1+\eta) .
$$

The condition for the lines $A B$ and $A_{10} B_{10}$ to be parallel again holds because it contains $p_{-}$as a factor. Since $A_{10} B_{10} C_{10} D_{10} E_{10} F_{10} G_{10}$ is obviously a regular heptagon it follows that its sides are parallel with the corresponding sides of $A B C D E F G$.

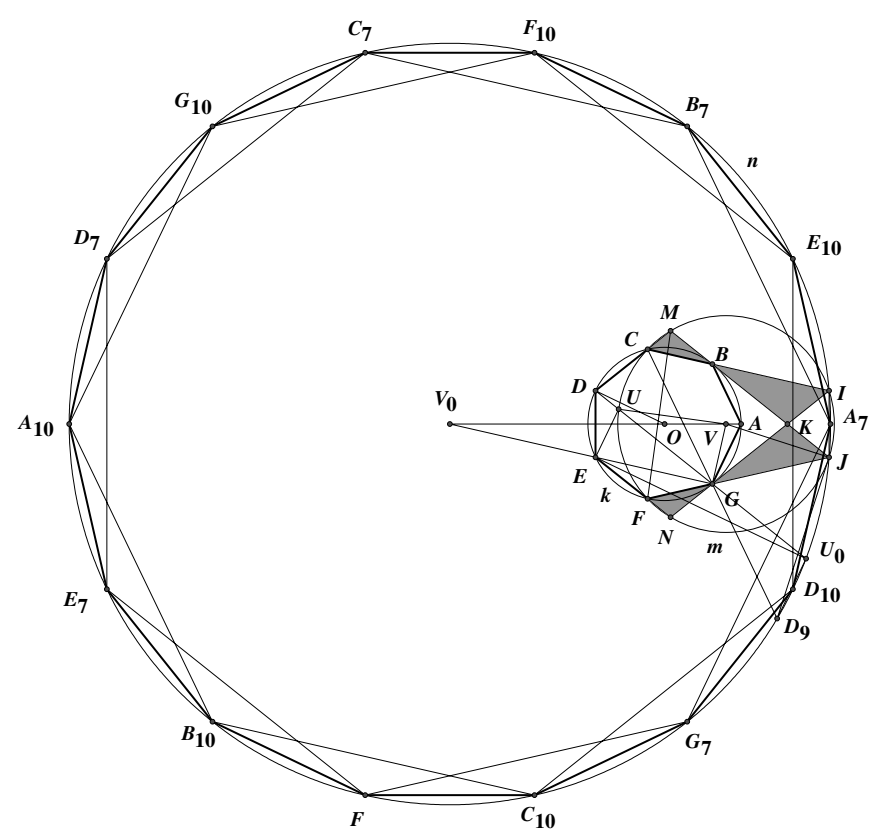

Fig. 10 Two regular heptagons on midpoints of shorter arcs inscribed in the circle $n$ and the regular 14-gon from their vertices (Theorems 8-11)

Theorem 11. The points $A_{7}, D_{10}, G_{7}, C_{10}, F_{7}, B_{10}, E_{7}, A_{10}, D_{7}, G_{10}, C_{7}, F_{10}, B_{7}, E_{10}$ are the vertices of the regular 14-gon (see Fig. 10).

Proof. Since $A_{10}=f\left(D_{7}-V_{0}\right)+V_{0}$, it follows that by rotating $D_{7}$ for the angle of $\frac{\pi}{14}$ radians we get $A_{10}$. This implies the claim of the theorem. Notice that the regular heptagons $A_{7} B_{7} C_{7} D_{7} E_{7} F_{7} G_{7}$ and $A_{10} B_{10} C_{10} D_{10} E_{10} F_{10} F_{10}$ are symmetric with respect to the perpendicular at $O$ to the line $O A$.

\section{References}

[1] Bankoff, L.; Garfunkel, J.: The heptagonal triangle. Math. Mag. 46 (1973), 163-187.

[2] Čerin, Z.: Geometrija pravilnog sedmerokuta. PlayMath (Časopis za matematiku i informatiku V. gimnazije u Zagrebu), br. 2 (2003), 22-28. (in Croatian).

[3] Čerin, Z.: On geometry of the regular heptagon. J. Geom. and Graphics 9 (2005), 119-132.

[4] Čerin, Z.: Regular heptagon's midpoints circle. Sarajevo J. Math. 2 (14), no. 1, (2006), 119-131. 
[5] Deaux, R.: Introduction to the geometry of complex numbers. Ungar Publishing Co., New York 1956.

[6] Hahn, L.: Complex numbers and geometry. Mathematical Association of America, Washington 1994.

[7] Morley, F.; Morley, F.V.: Inversive Geometry. Chelsea Publ. Co., New York 1954.

[8] Pedoe, D.: A course of geometry. Cambridge University Press, Cambridge 1970.

[9] Schwerdtfeger, H.: Geometry of complex numbers. Oliver and Boyd, Toronto 1962.

[10] Yaglom, I.M.: Complex numbers in geometry. Academic Press, New York 1968.

\section{Appendix}

This note is an example of a new approach to geometry offered by computers. In this appendix we will reveal how one can check our results on a computer.

The figures are made in the software The Geometer's Sketchpad that could also be used for approximate verification of statements and in the discovery of new theorems about geometric objects like regular heptagons.

Our mathematically correct proofs where realized on a computer in the software Maple V (version 8). We will describe how to prove Theorems 1-3 in Maple V.

First we give points as ordered pairs $[p, q]$ of a complex number $p$ and its conjugate $q$. The complex number $f$ is the $14^{\text {th }}$ root of unity.

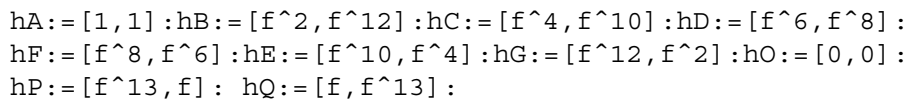

Here we use hA instead of $A$ as a name of the first vertex because with plain letters we run into problems as some letters are reserved in Maple $\mathrm{V}$ (for example D).

We introduce the shortening FS for the simultaneous use of commands factor and simplify to reduce typing.

$$
\text { FS: }=x->\text { factor }(\operatorname{simplify}(x)):
$$

The following function computes the square of the distance between two points $a$ and $x$.

$$
\mathrm{di}:=(\mathrm{a}, \mathrm{x})->\operatorname{FS}((\mathrm{a}[1]-\mathrm{x}[1]) *(\mathrm{a}[2]-\mathrm{x}[2])):
$$

Lines are represented as ordered triples $[u, v, w]$ of coefficients of their equations $u z+v \bar{z}+w=0$. The function $l i$ gives the line through two different points.

$$
1 i:=(a, b)->\operatorname{FS}([a[2]-b[2], b[1]-a[1], a[1] * b[2]-a[2] * b[1]]):
$$

The function ins gives the intersection of two lines. (The names in and int are reserved!). When its usage results in the error message

$$
\text { Error, numeric exception: division by zero }
$$

then the lines are parallel (when they do not have an intersection).

$$
\begin{array}{r}
\text { ins : }=(\mathrm{p}, \mathrm{q})->\mathrm{FS}([(\mathrm{p}[2] * \mathrm{q}[3]-\mathrm{p}[3] * \mathrm{q}[2]) /(\mathrm{p}[1] * \mathrm{q}[2]-\mathrm{p}[2] * \mathrm{q}[1]), \\
(\mathrm{p}[3] * \mathrm{q}[1]-\mathrm{p}[1] * \mathrm{q}[3]) /(\mathrm{p}[1] * \mathrm{q}[2]-\mathrm{p}[2] * \mathrm{q}[1])]):
\end{array}
$$

This short introduction into analytic plane geometry via complex numbers concludes with the simple functions for the midpoint of two given points and the parallel and the perpendicular through a given point to a given line.

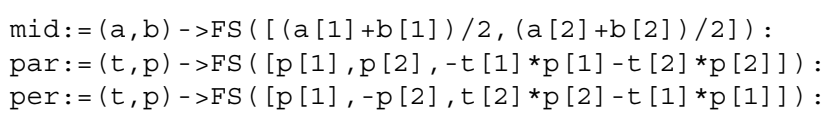


The points $U$ and $V$ are now obtained as follows:

$$
h U:=i n s(l i(h C, h E), l i(h D, h G)): h V:=i n s(l i(h B, h P), l i(h G, h Q)):
$$

The circle $m$ is the locus of all points whose square of distance to the point $V$ is equal to 2 . The following function $\mathrm{hm}$ associates to a point the difference of the square of its distance from $V$ and 2. A point $T$ will lie on the circle $m$ if and only if the value $h m(T)$ is zero.

$$
\mathrm{hm}:=\mathrm{x}->\mathrm{FS}(\mathrm{di}(\mathrm{x}, \mathrm{hV})-2):
$$

We check now the values of $h m$ in the points $C, F$, and $U$

$\mathrm{hm}(\mathrm{hC}) ; \mathrm{hm}(\mathrm{hF}) ; \mathrm{hm}(\mathrm{hU})$;

The output for the first two inputs is $p_{-} K$ where $K$ is

$$
f^{18}-f^{17}-f^{15}+f^{14}-f^{13}+f^{12}-2 f^{11}+f^{10}-f^{9}+3 f^{8}+f^{7}-f^{5}+f^{4}-2 f-2,
$$

while for the third it is $\frac{p_{-} M}{N^{2}}$ where $N=\left(f^{2}+f+1\right)\left(f^{2}-f+1\right)$ and

$$
\begin{aligned}
M= & -2-2 f-9 f^{19}-3 f^{11}-4 f^{3}-7 f^{5}-10 f^{15}+11 f^{14} \\
& -11 f^{17}+9 f^{16}-3 f^{23}-6 f^{21}-4 f^{2}-f^{25}+f^{26}+9 f^{10}-4 f^{9} \\
& +4 f^{8}-5 f^{7}-f^{6}+7 f^{18}+5 f^{20}+4 f^{22}+3 f^{24}-5 f^{4}+13 f^{12}-7 f^{13} .
\end{aligned}
$$

Since all of these expressions contain $p_{-}$as a factor we infer that they are equal to zero.

The points $I, N, J, M$, and $K$ are defined as follows:

$$
\begin{aligned}
& h I:=i n s(l i(h B, h C), \operatorname{per}(h G, l i(h G, h O))): \\
& h N:=i n s(l i(h E, h F), \operatorname{per}(h G, l i(h G, h O))): \\
& h J:=i n s(l i(h F, h G), \operatorname{per}(h B, l i(h B, h O))): \\
& h M:=i n s(l i(h C, h D), \operatorname{per}(h B, l i(h B, h O))): \\
& h K:=i n s(l i(h I, h N), l i(h J, h M)):
\end{aligned}
$$

We compute the values of $h m$ in the points $I, N, J$, and $M$ to verify that they lie on the circle $m$. Next we find the midpoint of the segment $K O$ and show that it is at the distance zero from the point $V$.

$\mathrm{hm}(\mathrm{hI}) ; \mathrm{hm}(\mathrm{hN}) ; \mathrm{hm}(\mathrm{hJ}) ; \mathrm{hm}(\mathrm{hM}) ; \mathrm{di}(\mathrm{hV}, \operatorname{mid}(\mathrm{hK}, \mathrm{kO}))$;

For the last claim we will use the following functions that test if two triangles are directly or reversely similar (see [5]).

$$
\begin{aligned}
& \operatorname{sid}:=((a, b, c),(p, q, r))->F S(a[1] * q[1]-a[1] * r[1]-b[1] * p[1]+ \\
& \mathrm{b}[1] * r[1]+\mathrm{c}[1] * \mathrm{p}[1]-\mathrm{c}[1] * \mathrm{q}[1]): \\
& \operatorname{sir}:=((a, b, c),(p, q, r))->F S(q[2] * a[1]-r[2] * a[1]-p[2] * b[1]+ \\
& r[2] * b[1]+c[1] * p[2]-q[2] * c[1]): \\
& \operatorname{sid}((h D, h E, h G),(h J, h K, h G)) ; \operatorname{sid}((h D, h E, h G),(h F, h N, h G)) ; \\
& \operatorname{sir}((h D, h E, h G),(h I, h K, h B)) ; \operatorname{sir}((h D, h E, h G),(h C, h M, h B)) \text {; }
\end{aligned}
$$

\section{Zvonko Čerin}

Kopernikova 7

10010 Zagreb, Croatia

e-mail: cerin@math.hr 